\title{
Dynamics of Internal Electric and Phonon Fields in n-GaAs Pumped with Ultrashort Pulses
}

\author{
Fabrício M. Souza and J. Carlos Egues \\ Departamento de Física e Informática, Instituto de Física de \\ São Carlos, Universidade de São Paulo, \\ 13560-970 São Carlos,São Paulo, Brazil \\ Received February, 1998
}

\begin{abstract}
We investigate the ultrafast dynamics of an electron-hole plasma coupled to phonons in bulk GaAs excited with femtosecond laser pulses. Our approach is based on balance equations directly derived from the Boltzmann equation within the relaxation-time approximation. Poisson's equation together with a phenomenological driven-harmonic-oscillator equation supplements our description by accounting for time-dependent electric and vibrational fields. Our calculated internal fields show oscillations at frequencies characteristic of those of coupled plasmon-phonon modes. Our results are consistent with recent experimental data.
\end{abstract}

Above-band-gap optical excitation of semiconductors generates highly non-equilibrium photocarrier distributions. With the advent of femtosecond laser pulses and the subsequent development of pump-and-probe spectroscopies, researchers have been able to probe the ultrafast/transient dynamics of these photocarriers with subpicosecond time resolution. A variety of new physical phenomena are observed at these ultrashort timescales[1].

More specifically, pump-and-probe measurements in GaAs systems have found strong modulation of the optical reflectivity at frequencies of coupled plasmoncoherent-phonon modes.[2] Mode beating is also observed. The modulation of the optical signal reflects the coupled dynamics of the internal electric and phonon fields following the pump excitation; these time-dependent fields modify the reflectivity of the sample via the electro-optic effect.

Here we investigate the ultrafast internal-field dynamics of a photocreated electron-hole plasma coupled to a vibrational mode in bulk GaAs. The time evolution of the photocarrier densities and currents is described semiclassically in terms of moments of the Boltzmann equation. Coherent-phonon effects are accounted for by considering a phenomenological driven-harmonicoscillator equation which is coupled to the electron-hole plasma via Poisson's equation[3].

Our numerical results for the time evolution of internal fields, particle densities, and currents, display interesting oscillations characteristic of coupled plasmonphonon modes. Mode beating is also present in our simulations at high excitation densities. These features are consistent with recent data.

Model. We assume the photogenerated electrons and holes belong to parabolic bands with isotropic effective masses $m_{e}^{*}=0.067 m_{0}$ and $m_{h}^{*}=0.52 m_{0}$, respectively. We consider motion only perpendicular to the surface of the GaAs, i.e., our model is one dimensional. The electron and hole photoplasmas are assumed to be in quasi-equilibrium at constant temperatures ("hot-carrier regime"[1]) $T_{e}$ and $T_{h}$, respectively, different from the lattice temperature.[4] With these assumptions we derive hydrodynamics equations - moments of the Boltzmann equation - for electron and hole particle densities and their corresponding current densities. These are supplemented with an electric-field driven harmonic-oscillator equation and Poisson's equation which effectively provides a coupling among electrons, holes, and phonons. Damping of both vibrational and electronic coordinates are included in our description through appropriate relaxation times. We assume a generation rate which is Gaussian in time and exponentially decreasing, with a absorption length $\alpha$, away from the surface. Altogether we have a set of five coupled partial differential equations - two of which are non-linear - plus Poisson's equation.[5] A final ingredient in our description is the inclusion of depletion-layer effects.

Depletion layer. Both doped and unintentionally doped GaAs samples present a "surface electric field" or depletion-layer electric field due to Fermi-level pinning. We model depletion-layer effects in a simplistic 
fashion. In $n$-GaAs, we take the depletion-layer electric field as the resulting screened field obtained by allowing the electrons to screen a constant electric field $E_{0}$ applied across the system. The magnitude of this constant field at the surface is a parameter in our model. Our simulations use this initial configuration of field (and the corresponding phonon field and electron density) to determine the time evolution of the system under laser excitation. This crude way to include depletion-layer effects is not crucial to our qualitative analysis.

Our numerical results[6] in Figs. 1(a) and 1(b) are obtained for a $4 \mu \mathrm{m}$ thick $n$-GaAs sample with $n=3.0 \times 10^{17} \mathrm{~cm}^{-3}$; the excitation density is $n_{e x c}=$ $1.0 \times 10^{18} \mathrm{~cm}^{-3}$. We assume the momentum relaxation time to be the same for both electrons and holes, $\tau_{e}=\tau_{h}=100 \mathrm{fs}$; the relaxation-time for phonons $\tau_{p h}$ (anharmonic effects) is taken to be $4 \mathrm{ps}$. We use $E_{0}=-20 \mathrm{kV} / \mathrm{cm}$ and excess energies for the photocarriers corresponding to $T_{e} \sim 387 \mathrm{~K}$ and $T_{h} \sim 49 \mathrm{~K}[2]$; at these low excitation enegies, carrier transfer to other valleys $(X$ and $L$ ) can be neglected.
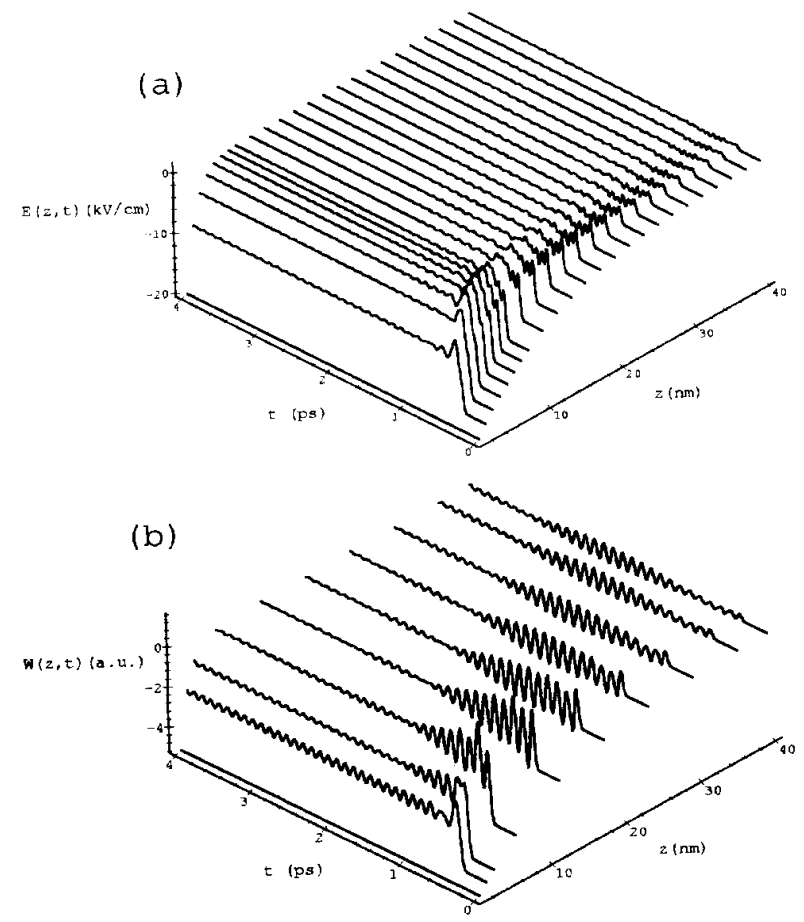

Figure 1. Internal electric- and phonon-field landscapes, (a) and (b), respectively, in $n$-doped GaAs system excited with an ultrashort pulse peaked at $0.3 \mathrm{ps}$. The sudden screening of the depletion-layer electric field $[E(z, 0)$ profile] drives characteristic coupled oscillations in the internal fields. Further analysis indicates that these oscillations occur at frequencies of local plasmon-phonon coupled modes. Mode beating is also seen in (a) and (b).

Figure 1(a) displays the internal electric-field landscape $E(z, t)$ in an $n$-GaAs system following an aboveband-gap optical excitation by a $50-$ fs pulse laser. The $t=0$ electric-field profile $E(z, 0)$ represents the depletion layer-field used in the simulation. Deviations from this profile only occurs at around $t=0.3 \mathrm{ps}$ when the generation of photocarriers by the pulse takes place.

Particularly interesting are the oscillations in the internal electric field. They are more pronounced near the surface, where the depletion-layer field is stronger, and around the pulse peak at $0.3 \mathrm{ps}$. These oscillations correspond to local coupled plasmon-phonon modes launched by the ultrafast screening of the depletionlayer field[7]. This is readily verified by Fourier transforming the field in Fig. 1(a) at different positions. Interestingly enough, we find that these oscillations are still preserved in the Fourier spectrum of the integrated field. This "average spectrum" should be a more physical quantity to relate to data; experiments probe volumar field changes through the electro-optic effect.

Figure 1(b) shows the "normalized lattice displacement" $W(z, t)[8]$. Observe that the lattice is initially in a "stretched" equilibrium configuration at $t=0$ due to the non-zero depletion-layer field. The photocarrierinduced screening of the depletion-layer electric field drives lattice vibrations; these lattice oscillations also alter the electric field via Poisson's equation - which includes lattice polarization charges. Mode beating is observed in both 1(a) and 1(b)

We have briefly presented a theoretical investigation of the internal-field dynamics in $n$-doped GaAs systems following an ultrashort pulse excitation. The internal electric and phonon fields are relevant quantities directly related to the optical reflectivity of the system via the electro-optic effect. Within the hot-carrier regime, our approach describes the dynamics of the electron and hole photoplasmas in terms of balance equations for particle and current densities. Poisson's equation provides the coupling among electrons, holes and phonons. We find internal-field landscapes modulated by noticeable oscillations. Further analysis (Fourier spectrum of the fields) indicates that these oscillations are related to local plasmon-phonon coupled modes of the system. Mode beating is also seen. The above features are consistent with recent data[2].

This work has been supported by Fundação de Amparo à Pesquisa do Estado de São Paulo (FAPESP). F.M.S. gratefully acknowledges a fellowship from FAPESP. J.C.E. thanks A.V. Kuznetsov, T. Dekorsy, and G.C. Cho for useful discussions.

\section{References}

[1] For a recent review on ultrafast phenomena, see J. Shah, Ultrafast Spectroscopy of Semiconductors and 
Semiconductor Nanostructures, Springer Ser. SolidState Sci., Vol. 115 (Springer, Berlin, Heidelberg 1996).

[2] M. Hase, K. Mizoguchi, H. Harima, F. Miyamaru, S. Nakashima, R. Fukasawa, M. Tani, and K. Sakai, J. Lumin. 76\&77, 68 (1998); G. C. Cho, T. Dekorsy, H. J. Bakker, R. Hövel, and H. Kurz, Phys. Rev. Lett. 77, 4062 (1996).

[3] A. V. Kuznetsov and C. J. Stanton [Phys. Rev. B 51, 7555 (1995)] have also studied the dynamics of internal fields in a GaAs systems. Our treatment extends theirs. In our approach, inhomogeneous density effects (drift and diffusion away from the surface) are accounted for in our balance equations, for the current and particles densities, Poisson's equation, and the harmonicoscillator equation.

[4] Carrier-carrier scattering is very effective in redistributing the excess energies of the electrons and holes, within each photoplasma, respectively; this thermalization occurs within tens of femtoseconds. See C. W.
W. Bradley, R. A. Taylor, and J. F. Ryan, Solid State Electron. 32, 1173 (1989).

[5] A more detailed description of our model and results, including the equations, will published elsewhere.

[6] We solve our coupled equations using standard numerical schemes for the propagation of partial differential equations; see, W. H. Press, S. A. Teukolsky, W. T. Vetterling and B. P. Flannery, Numerical Recipes in $C$, the Art of Scientific Computing, (Cambridge University, London, 1996) $2^{\text {nd }}$ edition, Ch. 19.

[7] Diffusion of photocarriers, although included in our model, is not relevant since the absortion length is about $1000 \mathrm{~nm}$ at the pump energy considered $(\sim 1.467$ $\mathrm{eV})$.

[8] See Max Born and Huang Kun, Theory of Crystal Lattices, The International Series of Monographs on Physics, (Oxford University Press, Oxford, 1985), $2^{\text {nd }}$ edition, Ch. II, p. 104. 